\title{
Eventos adversos no cuidado da criança: concepções de familiar/cuidador na atenção básica
}

\author{
Adverse events in the health care for children: conceptions of family/caregiver \\ in the primary care
}

Mariana Ferreira Arrieche Lopez ${ }^{1}$, Wiliam Wegner ${ }^{2}$

\begin{abstract}
RESUMO
Objetivo: Identificar os eventos adversos no cuidado da criança na atenção básica à saúde sob a ótica do familiar/cuidador e analisar os eventos adversos a partir dos pressupostos da segurança do paciente.

Materiais e Métodos: Pesquisa qualitativa do tipo exploratório-descritiva desenvolvida em Unidade Básica de Saúde de Porto Alegre/RS. Onze (11) familiares/cuidadores participaram por meio de uma entrevista semiestruturada, a qual foi submetida à análise temática. O projeto foi aprovado pelo Comitê de Ética em Pesquisa da Secretaria Municipal de Saúde de Porto Alegre/RS.

Resultados: No processo de análise emergiram três categorias: os eventos adversos nas ações de cuidado em saúde; a garantia de acesso ao serviço e a resolutividade como princípio para segurança do paciente; e a qualificação do profissional, competências e habilidades como pressupostos para segurança do paciente.

Conclusão: A qualificação do profissional, a resolutividade e a garantia de acesso são pressupostos para a segurança do usuário na atenção básica à saúde.
\end{abstract}

Palavras-chave: segurança do paciente; criança; atenção básica à saúde; cuidado da criança.

\begin{abstract}
Objective: To identify child care adverse events in the primary health, from the perspective of the family/caregiver and to analyze adverse events from the assumption of patient safety.

Materials and Methods: It is a qualitative exploratory descriptive research developed in the Basic Health Unit in Porto Alegre/RS. Eleven (11) family members/caregivers participated in this study through a semi-structured interview, which was submitted to a thematic analysis. The project was approved by the Ethics Committee of the Municipal Health Secretariat.

Results: The process of analysis showed three categories: adverse events in health care actions; guarantee of access to the health service and the resolvability as a principle for patient safety; and professional qualification, skills and abilities as prerequisites for patient safety.

Conclusions: The qualification of the professional, the resolvability and the guaranteed access are assumptions to the user's safety in primary health care.
\end{abstract}

Keywords: patient safety; child; primary health care; child care.

\footnotetext{
${ }^{1}$ Enfermeira. Especialista em Saúde da Criança e do Adolescente pelo Programa de Residência Multiprofissional e em Área Profissional da Saúde PREMUS/HSL(PUCRS). Enfermeira da Unidade de Internação Pediátrica do Hospital São Lucas da PUCRS.

${ }^{2}$ Enfermeiro. Doutor em Enfermagem. Membro da Rede Brasileira de Enfermagem e Segurança do Paciente (REBRAENSP). Professor Adjunto do Departamento de Enfermagem da Universidade Federal de Ciências da Saúde de Porto Alegre (UFCSPA).
} 


\section{INTRODUÇÃO}

A preocupação com a segurança do paciente surgiu no final da década de 1990 com a publicação do relatório To Err is Human: Building a safer health care system, o qual revelou que aproximadamente $44 \mathrm{mil} \mathrm{a}$ 98 mil pessoas morriam por ano nos Estados Unidos devido a erros relacionados com a assistência à saúde ${ }^{1}$. Em 2001, a Academia Americana de Pediatria (AAP) recomendou alguns princípios para a segurança do paciente como: desenvolver estratégias com ações focadas na organização, nos profissionais e nos usuários; os serviços devem particularizar os cuidados à criança, entre outros ${ }^{2}$.

As causas dos erros e eventos adversos são multifatoriais, assim, os profissionais de saúde estão suscetíveis a cometer eventos adversos, quando os processos técnicos e organizacionais são complexos e/ou mal planejados ${ }^{3}$. Ao encontro do fortalecimento mundial do tema segurança do paciente, em 2004, foi lançada a primeira edição da Aliança Mundial para Segurança do Paciente, a qual se voltou para a criação e desenvolvimento de políticas e práticas em prol da segurança do paciente para todos os países membros da Organização Mundial da Saúde (OMS) ${ }^{4}$.

Em 2002, a 55 Assembléia Mundial de Saúde atribuiu à OMS a responsabilidade de estabelecer normas e padronizações, dando suporte aos países no desenvolvimento de políticas públicas e práticas de segurança do paciente ${ }^{5}$. Em 2005, com o tema "Clean Care is Safe Care" (Cuidado limpo é Cuidado seguro), o foco foram as infecções relacionadas com a assistência à saúde, uma das estratégias prioritárias foi a higienização das mãos como um primeiro passo de garantir elevados padrões de controle de infecção e segurança do paciente ${ }^{6}$. Ainda em 2009, o Desafio Global proposto pela Aliança Mundial para Segurança do Paciente foi "Safe Surgery Save Lives" (Cirurgia Segura Salva Vidas), tornando a cirurgia segura uma prioridade nas ações das instituições de saúde ${ }^{7}$. Em 2010, foi elaborada uma cartilha sobre os "10 Passos para a Segurança do Paciente" para contemplar os principais pontos de impacto na prática assistencial, descrevendo processos básicos de cuidado de enfermagem para a promoção da segurança do paciente, podendo inclusive subsidiar as ações na atenção básica à saúde ${ }^{8}$.

Entende-se por segurança do paciente a redução do risco de danos desnecessários a um mínimo aceitável. O incidente de segurança do paciente é um evento ou circunstância que poderia ter resultado ou que resultou em danos desnecessários para o paciente ${ }^{9}$. Já os eventos adversos são falhas no planejamento e/ou na execução de uma ação que têm objetivos e resultados esperados; nem todos os erros vão culminar em injúrias, as injúrias são os danos causados por um agente ou evento, configurando-se em evento adverso ${ }^{10-12}$.

Frente a este cenário, como a segurança do paciente é compreendida no contexto da atenção básica em saúde pelo familiar/cuidador da criança? Quando esse familiar/cuidador identifica momentos em que o cuidado em saúde pode representar risco para a saúde da criança? As questões desta pesquisa provocam reflexões e necessitam ser problematizadas no contexto de cuidado na atenção básica. Mediante a problemática apresentada, os objetivos deste estudo são: identificar os eventos adversos no cuidado da criança na atenção básica à saúde sob a ótica do familiar/cuidador e analisar os eventos adversos a partir dos pressupostos da segurança do paciente.

\section{MATERIAIS E MÉTODOS}

Trata-se de um estudo de abordagem qualitativa do tipo exploratório-descritivo, a abordagem qualitativa não pode pretender o alcance da verdade com o que é certo ou errado, deve preocupar-se com a compreensão da lógica que permeia a prática da realidade ${ }^{13}$. A pesquisa foi desenvolvida em uma Unidade Básica de Saúde de Porto Alegre/RS. A população envolvida neste estudo foram 11 familiares/cuidadores da criança em atendimento na Unidade Básica de Saúde no período entre agosto e setembro de 2009. Os participantes foram escolhidos de forma aleatória durante a espera pelo atendimento pediátrico naquela Unidade Básica de Saúde.

Foi utilizado o critério de saturação das informações que, após certo número de entrevistas, o entrevistador começa a ouvir relatos muito semelhantes, havendo uma rarefação de informações novas ${ }^{14}$. Os critérios de 
seleção para participação na pesquisa foram: ser responsável pela criança, maior de idade e acompanhar a criança nos atendimentos; estar a criança em acompanhamento periódico da sua saúde.

A coleta de dados foi realizada por meio de entrevista semiestruturada, com questões abertas aos participantes, as quais trazem como temática a segurança da criança e família na unidade básica de saúde, quais as medidas realizadas para alcançar esta situação, o que significa segurança na unidade básica de saúde e compreender como o familiar/cuidador da criança entende isto. Finalizada a coleta de dados, a análise das informações seguiu as etapas da análise de conteúdo do tipo temática ${ }^{15}$.

Foram elaboradas cinco questões norteadoras para realizar a entrevista semiestruturada, as quais abordavam as questões sobre a segurança do paciente, no caso, criança, dentro da Unidade Básica de Saúde (UBS). As questões norteadoras foram as seguintes: 1) Você acha que a UBS protege a saúde da criança e família e garante sua segurança?; 2) O que você compreende por segurança dentro da UBS?; 3) Os profissionais de saúde da UBS apresentam cuidados especiais na atenção à criança e à família? Eles tem habilidades e conhecimento sobre as características da criança que é levada a UBS?; 4) Quando você leva a criança à UBS, já houve situações que provocaram desconforto, intercorrências ou eventos adversos no cuidado da criança? e 5) O que você acha que pode ser feito para melhorar a segurança e proteção da criança na comunidade onde reside?

O projeto de pesquisa foi aprovado no Comitê de Ética em Pesquisa da Secretaria Municipal de Saúde, processo de $\mathrm{n}^{\circ}$ 001.035483.09.1. Foram respeitados preceitos da Resolução CNS 196/96 e todos os participantes assinaram o Termo de Consentimento Livre e Esclarecido. Para a preservação do anonimato, os participantes foram codificados por números (01-11) seguido da consoante ( $P=$ Participante) e os fragmentos de análise em Unidades de Sentido (US) conforme o processo de codificação.

Na UBS pesquisada, são desenvolvidas práticas de saúde como: acolhimento, atendimento em pediatria, ginecologia e clínica geral. Em relação a procedimento de cuidado em saúde são realizados: curativos, vacinas, medicações injetáveis, teste do pezinho, nebulizações e distribuição de medicamentos. Quanto a ações programáticas, são desenvolvidos os Programas: Nascer, Prá-crescer, Esperança, Pré-natal, Prá-nenê e Hiperdia (acompanhamento de hipertensos e diabéticos) e Programas para câncer de mama e colo de útero propostos pelo Ministério da Saúde. Nessa contextualização, identifica-se uma variedade de questões que podem apresentar relação com limitação no cuidado em saúde, quando os pressupostos da segurança do paciente não estão plenamente garantidos.

Os sujeitos da pesquisa foram 11 participantes (familiares/cuidadores) que estavam acompanhando a criança em atendimento à criança na Unidade Básica de Saúde. A idade das participantes variou entre 19 e 35 anos. Todos os participantes foram do sexo feminino, representados pela figura materna. A maior parte das participantes é solteira, possui mais de um filho, tem o ensino médio completo, sem renda mensal e residem com outros familiares.

\section{RESULTADOS E DISCUSSÃO}

A análise das informações empíricas obtidas junto aos participantes da pesquisa possibilitou a formulação de três categorias de análise, que foram: Os eventos adversos nas ações de cuidado em saúde; A garantia de acesso ao serviço e a resolutividade como princípio para segurança do paciente e A qualificação do Profissional: competências e habilidades como pressupostos para a segurança do paciente. Essas serão apresentadas e discutidas sob o prisma da segurança do paciente após a contextualização do cenário de pesquisa.

Os eventos adversos nas ações de cuidado em saúde

O profissional da saúde deve atuar nas ações de promoção, proteção e recuperação da saúde, garantindo a segurança do paciente, reduzindo ou eliminando o risco de danos desnecessários associados com a saúde a um mínimo aceitável, e assim evitando a ocorrência de eventos adversos no cuidado à saúde ${ }^{9}$. 
A prática do profissional de saúde pode gerar um quase incidente (near miss), um incidente que ainda não atingiu o paciente, um incidente sem dano. $O$ evento adverso vai ser um incidente com dano, ou seja, aquele que conduz dano para o paciente ${ }^{9}$.

O evento adverso na atenção básica pode estar relacionado tanto com a prática do profissional, quanto com a falta de informação sobre a saúde. Entretanto, é importante refletir que as falhas são sistêmicas e atreladas a processos, não ocorrendo isoladamente.

$\mathrm{Na}$ concepção dos familiar/cuidador os eventos adversos podem ocorrer em todas as etapas do atendimento à saúde, podem estar ligados a diversos fatores do processo de trabalho, tais como: falha nos cuidados prestados em procedimentos, falha na obtenção de informação, estresse, inexperiência, sobrecarga de trabalho, entre outros ${ }^{9}$.

Uma vez minha filha veio tomar vacina e depois ficou com o braço roxo. Acho que aplicaram mal. (P9US49)

Comentei com o enfermeiro que não podia colocar pomada no pé do meu filho, onde ele tinha uma ferida, porque estava cicatrizando, e ele disse que podia sim. Daí colocou e infeccionou aquele ponto. (P4US23)

Dever-se-ia repensar as condutas e entender que esses eventos adversos podem gerar transtorno, dano ou prejuízo para o ser humano no cuidado em saúde, e também pode gerar insegurança por parte do familiar/cuidador, muitas vezes dificultando a procura pelo serviço de saúde, pois a relação de confiança não subsidia suas necessidades.

Uma questão chave que tem impacto na segurança do paciente é o número insuficiente de profissionais de saúde qualificados em todo o mundo ${ }^{15}$. No momento atual, os profissionais da saúde deveriam desenvolver iniciativas para evitar, prevenir e reduzir eventos adversos, promovendo a segurança do paciente e assumindo que falhas acontecem ${ }^{4}$. Todos os profissionais poderiam desenvolver uma atitude proativa em frente da segurança do paciente que por eles será cuidado.

A metodologia do acolhimento dos usuários, por exemplo, envolve questões relacionadas diretamente com o modo de atuar do profissional de saúde, é uma metodologia de atendimento que pressupõe empatia, afeto, preocupação, vínculo entre outros, isto pode garantir a integralidade da atenção em saúde e a qualidade da assistência prestada.

Deve-se garantir a existência de mecanismos para prevenção e minimização de erros, visando à promoção da segurança do paciente e o estabelecimento da comunicação entre a equipe, os pacientes $e$ as instituições ${ }^{16}$.

A atenção básica pode gerar inúmeros eventos adversos, o principal pode ser o distanciamento com 0 usuário que perde a credibilidade e não procura esse serviço. Isso vai culminar em superlotação e sobrecarga nos hospitais, o que pode ser considerado um evento adverso, pois gera danos aos usuários que têm o acesso dificultado.

A garantia de acesso ao serviço e a resolutividade como princípio para segurança do paciente

Quando procuramos o serviço de saúde com o intuito de receber atendimento que resolva uma necessidade de saúde, partimos com vontades pré-determinadas como, por exemplo, um atendimento satisfatório que resolva o problema daquele momento.

O acesso aos serviços de saúde é um direito de todo o cidadão brasileiro, garantido na Lei 8.080 do SUS (Sistema Único de Saúde), e pelas políticas públicas de saúde. Entretanto, visualizam-se inúmeros problemas relacionados com a atenção em saúde oferecida, entre eles, o acesso ao serviço tem destaque. No Estatuto da Criança e do Adolescente é assegurada, por intermédio do Sistema Único de Saúde, a garantia de acesso universal e igualitário às ações de saúde e serviços de promoção, proteção e recuperação da saúde a todas as crianças e adolescentes ${ }^{16}$.

A universalidade de acesso garante o acesso e atendimento a toda a população, porém na prática observa-se que cada serviço interpreta de maneira diferente este princípio. Os pesquisados identificam isso nos relatos a seguir:

Se eu venho consultar com mais de um filho, tenho que marcar um dia para cada filho, isso me incomoda. É 
uma senha por dia. (P1US1)

Uma vez tive um problema de saúde com meu filho e não puderam me atender porque eu não tinha marcado ficha, e era uma urgência. (P2US12)

A facilidade de acesso propicia a satisfação do usuário com o atendimento, determinando a escolha do serviço de saúde e estabelecendo frequentemente um bom vínculo ${ }^{17}$. Assim, a facilidade na busca pelo serviço e a satisfação dos usuários no atendimento seriam uma estratégia para minimizar os eventos adversos e consequentemente uma ação de segurança para o paciente.

Cada usuário tem sua percepção para avaliar a qualidade do atendimento de saúde, mas existem questões que não se modificam. A resolutividade dos serviços de saúde é uma maneira de avaliação a partir dos resultados obtidos no atendimento ${ }^{18}$. O familiar/cuidador demonstra compreensão sobre onde e o que é importante para um serviço de atenção primária.

\section{Resolvo o problema no posto. (P1US6)}

Tem que fazer algo sobre as doenças respiratórias. (P5US32)

Mesmo que o atendimento tenha sido satisfatório e tenha atendido às necessidades do usuário naquele momento, notamos que ainda existem ações a serem desenvolvidas para a resolutividade atingir não apenas o atendimento no serviço, mas também a continuidade do cuidado em outro cenário.

Toda a população deve ter a garantia de acesso ao serviço de saúde, com o intuito de atender as suas necessidades de saúde, desde o atendimento inicial até a conclusão e avaliação final, ou seja, a resolução da causa que levou a criança a ser levada ao serviço. Isso pode garantir a segurança da criança, pois seus problemas são resolvidos no primeiro nível de atenção.

\section{A Qualificação do Profissional: competências e} habilidades como pressupostos para a segurança do paciente

Atualmente, percebe-se que a qualificação dos profissionais de saúde é um tema em discussão, principalmente pelo aumento da oferta de novos profissionais e também de centros de formação. Isto contribui para a fragmentação e superficialidade no ensino-aprendizado. Diante disso, identificam-se lacunas em vários aspectos importantes para o exercício profissional nas práticas, entre elas, destacamse as relações interpessoais, a empatia, a capacitação técnica e a fundamentação teórica, entre outras.

As ações para melhorar a segurança da criança e família vão além das práticas de saúde, iniciam-se pela conduta dos profissionais. O depoimento a seguir contextualiza uma realidade comum no serviço investigado.

Acho que os funcionários poderiam gritar menos, serem mais educados, e cuidar o que falam. (P10US58)

Apesar disso, os usuários acreditam que é possível ter segurança se houver melhora nas relações entre os profissionais e, principalmente, na receptividade da demanda que chega ansiosa e necessitada por uma escuta.

O trabalho em equipe viabiliza a qualidade do atendimento, buscando diferentes conhecimentos, experiências e práticas, mas somente essas características não são suficientes para qualificar a assistência em saúde, se não existe uma comunicação e interação entre a equipe de saúde e também entre os usuários. Assim como o trabalho multidisciplinar que traz diferentes conhecimentos, opiniões e concepções gerando uma diversidade de olhares frente aos problemas de saúde dos usuários.

O profissional de saúde costuma valer-se das relações de poder e dominação em relação aos usuários de um serviço de saúde ${ }^{19}$. Na concepção do familiar/cuidador, as relações necessitam ser próximas e seguras.

\section{Ninguém informa nada. (P2US10)}

Ás vezes parece que não sabem o que estão fazendo. (P10US50)

A conduta da equipe é essencial para a segurança do usuário, muitas vezes os profissionais acabam tendo 
condutas e posturas insatisfatórias, podendo predispor a ocorrência de eventos adversos. Para evitar riscos ou danos aos envolvidas, sejam usuários, profissionais, alunos dos serviços de saúde, é necessário respeitar a sua própria autonomia e a do outro, isso evitaria danos ao processo educativo/assistencial ${ }^{20}$.

A qualificação do profissional de saúde na atenção básica sugere o aperfeiçoamento de habilidades e competências, para que o cuidado seja significativo e resolutivo. Todavia, é essencial minimizar os conflitos e as divergências entre o fazer profissional.

Quando não consegue atingir seus objetivos na busca pelo serviço de saúde, o usuário acaba por tornar-se insatisfeito com o local e mais vulnerável a eventos adversos, muitas vezes essa insatisfação ocorre na espera por atendimento, gerando uma insegurança que pode desfazer a relação de confiança, que é necessária na atenção básica à saúde.

\section{CONSIDERAÇÕES FINAIS}

A atenção básica à saúde deveria ser o contato preferencial dos usuários com o serviço de saúde, ou seja, a primeira opção na busca por atendimento à saúde. Para que isso aconteça, é indispensável à satisfação do usuário com o serviço de saúde. Evidenciamos que o familiar/cuidador da criança identifica fatores que predispõem a ocorrência de eventos adversos, interferindo na segurança do paciente, desde o acesso ao serviço até o momento em que o usuário deixa o serviço. Eles compreendem que o cuidado seguro não deve por em risco a saúde e segurança daquele que está sendo atendido.

Algumas das implicações na procura pelo serviço de saúde trazem a temática da segurança do paciente em sua discussão, como a acessibilidade ao serviço de saúde, os familiares/cuidadores identificam a dificuldade em garantir acesso ao serviço de saúde e, em decorrência disto, acabam procurando os serviços de maior complexidade muitas vezes por problemas, que poderiam ser solucionados na atenção básica à saúde, isto interfere na resolutividade do serviço e não garante a segurança do paciente.

Em outro cenário, temos o acesso ao serviço de saúde garantido, mas a conduta do profissional de saúde gera desconfortos, insatisfação, e risco para a segurança do paciente, assim entramos na discussão das habilidades e competências dos profissionais de saúde no cuidado à criança e, com isto, podemos abranger a discussão para a formação em saúde. A falta de habilidade e competências é vista como fator de risco para ocorrência de eventos adversos, interferindo assim na segurança do paciente.

Os profissionais deveriam estar em constante atualização de suas práticas e poderíamos pensar que muitas vezes essa qualificação deveria ser dirigida à demanda do serviço de saúde, o que acabaria qualificando o relacionamento do profissional com o usuário com o objetivo de qualificar o atendimento de saúde, gerando satisfação para o usuário.

Todas estas questões nos levariam a alcançar um atendimento integral e seguro em que a família é cuidada em todas as suas interfaces. Assim, seria necessário desenvolver estratégias voltadas para esta realidade. A noção de eventos adversos incorpora uma visão crítica e ampliada sobre as dificuldades que 0 setor saúde vem deparando-se há anos. Acredita-se ser necessário incorporar os pressupostos da segurança do paciente em todos os níveis de atenção à saúde, especialmente nas ações da atenção primária à saúde.

\section{REFERÊNCIAS}

1. Kohn LT, Corrigan JM, Donaldson MS. To Err is Human: building a safer health care system. Washington (DC): National Academy Press; 2000.

2. American Academy of Pediatrics. Principles of patient safety in pediatrics. Pediatrics. 2001; 107(6):1473-5.

3. Silva AEBC. Segurança do paciente: desafios para a prática e a investigação de enfermagem. Rev Eletrôn Enferm [Internet]. 2010; 12(3):422. Disponível em: http://www.fen.ufg.br/revista/v12/n3/v12n3a01/htm.

4. World Health Organization. World Alliance for Patient Safety. Forward Programme 2008-2009. Geneva (Switzerland): World Health Organization; 2008.

5. Conselho Regional de Enfermagem do Estado de São Paulo - COREN/SP. 55ํㅗㄹ Assembléia da Saúde Mundial, 2002. Disponível em: http://inter.coren-sp.gov.br/sites/ default/files/cirurgia-segura.pd

6. World Health Organization. Clean Care is Safe Care, 2005. Geneva (Switzerland): World Health Organization; 2005. Disponível em: http://www.who.int/gpsc/en/

7. World Health Organization. Guidelines for Safe Surgery Save Lives. Tradução: Segundo desafio global para a segurança do paciente - OMS/Brasil. Geneva (Switzerland): World Health Organization. Disponível em: http://bvsms.saude.gov.br/bvs/publicacoes/seguranca pac iente_cirurgias_seguras_salva_vidas.pdf 
8. Conselho Regional de Enfermagem do Estado de São Paulo - COREN/SP; Rede Brasileira de Enfermagem e Segurança do Paciente. 10 Passos para a Segurança do Paciente, 2010. [Internet]. Disponível em: http://inter.coren-sp.gov.br/sites/default/files/ 10_passos_ seguranca_paciente.pdf

9. Runciman W, Hibbert P, Thomson R, Schaaf TVD, Sherman $H$, Lewalle AP. Towards an International Classification for Patient Safety: key concepts and terms. Int J Qual Health Care. 2009; 21(1):18-26.

10. World Health Organization. A World Alliance for Safer Health Care. More Than Words: Conceptual Framework for the International Classification for Patient Safety. Version 1.1. Final Technical Report. January 2009 Geneva (Switzerland): World Health Organization; 2009.

11. Cassiani SHB. A segurança do paciente e o paradoxo no uso de medicamentos. Rev Bras Enferm. 2005; 58(1):959.

12. Wegner W. A segurança do paciente nas circunstâncias de cuidado: prevenção de eventos adversos na hospitalização infantil [thesis]. Porto Alegre: Escola de Enfermagem /UFRGS; 2011.

13. Minayo MCS. O desafio do conhecimento: pesquisa qualitativa em saúde. São Paulo:Hucitec; 2007.

14. Costa AMN. O campo da pesquisa qualitativa e o método de explicitação do discurso subjacente. Psicol Refl Crit. 2007; 20(1):65-73.

15. Jha AK, Prasopa-Plaizier N, Larizgoitia I, Bates DW.
Patient safety research: an overview of the global evidence. Qual Saf Health Care. 2010; 19(1):42-7.

16. Brasil. Estatuto da Criança e do Adolescente: Lei n. 8.069, de 13 de julho de 1990. Lei n. 8.242 de 12 de outubro de 1991. 3aed. Brasília (DF): Câmara dos Deputados, Coordenação de Publicações; 2001.

17. Dall'Agnol CM, Lima MADS, Ramos DD. Fatores que interferem no acesso de usuários a um ambulatório básico de saúde. Rev Elet Enf. [periódico eletrônico]. 2009; 11(3): 674-80. Disponível em: http://www.fen.ufg.br/revista/ v11/n3/v11n3a27.htm.

18. Turrini RNT, Leitão ML, Cesar CLG. Resolutividade dos serviços de saúde por inquérito domiciliar: percepção do usuário. Cad Saúde Pública. 2008; 24(3):663-74.

19. Schatkoski AM, Wegner W, Alegri S, Pedro ENR. Segurança e Proteção à criança hospitalizada: revisão de literatura. Rev Latino-Am Enfermagem. 2009; 17(3):410-6.

20. Fernandes JD, Rosa DOS, Vieira TT, Sadigursky D. Dimensão ética do fazer cotidiano no processo de formação do enfermeiro. Rev Esc Enferm USP 2008; 42(2):396-403.

\section{Endereço para correspondência:}

Mariana Ferreira Arrieche Lopez Av. ljuí $n^{\circ} 350$, apto. 202

Porto Alegre/RS - CEP 90460-200

Telefone: +55 5193361742

E-mail: mfarrieche@gmail.com 